\title{
Reactive Power Optimization Based on Multi- agent System with Markov Game Theory
}

\author{
Weibao Zhang \\ Jinzhou Power Supply Branch \\ State Grid Liaoning Electric Power Supply Co. Ltd \\ Jinzhou, China \\ e-mail:zhangwb58@126.com
}

\author{
Zhongfei Gao \\ Jinzhou Power Supply Branch \\ State Grid Liaoning Electric Power Supply Co. Ltd \\ Jinzhou, China \\ e-mail:Zf562558@sohu.com
}

\begin{abstract}
This paper presents a distributed and reactive power optimization system based on Muti-Agent System framework. The various component and functions of monomer Agent are displayed in the optimization system. The collaboration and coordination between the Agent are achieved by information sharing through the communication. The dynamic contract net protocol which doped by three mental state performance parameters contains Agent, trust nodes, familiarity and integration introduced into the collaborative approach of Muti-Agent. The optimization process of lower voltage and reactive power are described based on the Dynamic contract net protocol under the smart grid environment. The Muti-Agent learning algorithm is proposed according to the influence of the chosen $Q$ value based on Markov game framework. The Reactive Agentbased design optimization process, management system architecture design, simulation protocol design are illustrated combined with Agent combines technical features and smart distribution network reactive power compensation behavioral characteristics.
\end{abstract}

Keywords-markov game;muti-agent; power systems ; reactive power optimization

\section{INTRODUCTION}

Reactive power optimization is the premise of meeting the safe operation of the power grid through transformers to adjust joints, the generator terminal voltage regulation and reactive power compensation device, such as switching to adjust the reactive power flow of scientific and rational distribution, to seek reasonable reactive power compensation point and the best compensation to achieve the balance of reactive power, improve voltage quality and reduce network losses and ensure the safe operation of the system to stabilize the economy. Traditional reactive power optimization is usually for a typical cross-section of the static load reactive power optimization, considering no load changes over time, but in reality, power load is constantly changing, static reactive power optimization does not meet the needs of grid operation, only as a reference, with some limitations. Dynamic Reactive Power Optimization is considered each time the load changes by adjusting the control equipment to optimize the operation of the program, making the system voltage of the highest quality over a period of time, the minimum power loss, and meet the operating constraints and control equipment conditioning system the number of restrictions. Traditional reactive power optimization methods include: linear programming method, Newton's method [1], interior point method [2]. In recent years, multi-agent systems, genetic algorithms [3], particle swarm optimization, ant colony algorithm, such as artificial intelligence algorithms from different ways to achieve the goal of optimizing reactive power, they have their own strengths, but also have their own flaws.

Multi Agent System (MAS) research is computer technology, control theory, artificial intelligence applications and other multi-disciplinary integration of technology research [4-9] .MAS research focus is to make the function independent agent (Agent) through consultation, coordination and collaboration, complex control tasks or solve complex problems. Strategies and learning is the internal mechanism Agent collaboration. Currently studying learning in Multi-Agent lack mature theory. Littman $[10,11]$ uses countermeasures as a MultiAgent Learning Framework, the Multi-Agent learning model into Markov measures proposed min-max-Q learning algorithm to solve the zero-sum Markov games, it is only suitable for handling competitive relationship between Agent according to need more Agent environment, MAS measures in the framework necessary to be able to make multiple Agent through collaboration and cooperation to accomplish a specific task, it must effectively address the competition with rivals Agent.

The study asked Muti-Agent based incentive learning theory principles to achieve optimal control of voltage and reactive power, structure and implementation of the framework established distribution network model of voltage and reactive power control systems, and global optimization model and the target voltage and reactive power control function analysis and improvement, solving distribution network in various situations and reactive power regulation mode, and in the case meet the system constraints, effectively improve network voltage quality and reduce power loss and achieve security, stability and economic operation of the system.

\section{SYSTEM FRAMEWORK}

This paper uses a distribution network reactive power and voltage Muti-Agent system based on centralized control method for distributed control strategy. The overall task for complete optimization and decomposition of mathematical methods Muti-Agent optimization techniques applied to optimize coordination with reactive power control, to achieve system voltage and reactive power optimal control and scheduling, to ensure the system global optimum. Muti-Agent system with internal autonomy, interaction, encapsulation and initiative and other characteristics, centralized distribution control 
system consists of a set of logically or physically Agent integral step of many reactive voltage Muti-Agent system based on their through collaboration to accomplish common tasks and by dividing a large complex system into smaller, communicate and coordinate with each other, and easy to manage system, implement complex power system reactive coordinated control, the structure is shown in Fig.1.

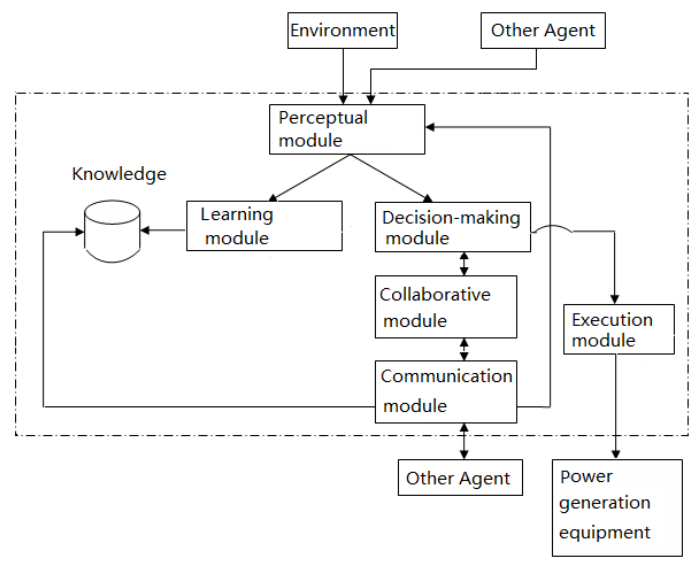

Figure 1. Muti-Agent Architecture

Full use of the autonomy, interaction, encapsulation and initiative Muti-Agent system to solve distribution network reactive power optimization problem of coordination and control, to ensure convergence speed, high accuracy, can be faster, more accurately converge to the global the optimal solution. This will be a complex distribution network reactive power optimization centralized coordination and control is divided into substep, the mutual coordination and control of Muti-Agent technology to communicate with each other, can be optimized in coordination with reactive power control to get better applications.

In this paper, based on voltage and reactive power distribution network coordinated control system MutiAgent theory hybrid structure, shown in Fig .2, where each individual Agent uses a hybrid model, with strong flexibility and quick response. The Muti-Agent system is divided into centralized control Agent, Distribution Agent and control node controls Agent, Agent centralized control reactive power in order to achieve optimal control of the whole system of centralized targets, distributed control Agent to achieve within the jurisdiction of the regional distribution of voltage and reactive power control target node Agent to complete the upper distribution optimization issued under the control of voltage and reactive power optimization Agent control commands.

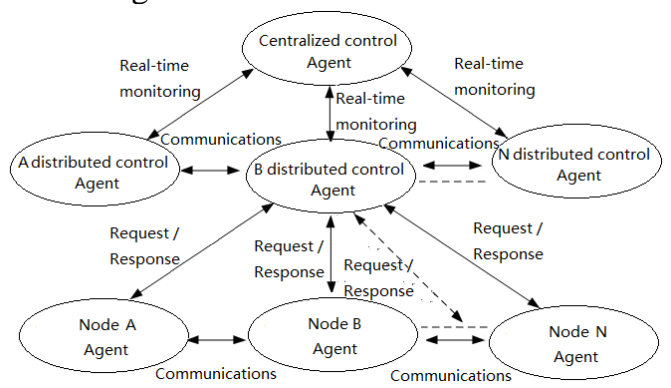

Figure 2. Control Systems
The system contains one or more centralized control Agent, Agent centralized control system for real-time monitoring under the jurisdiction of the members, combined with improved reactive nine field map and through various distribution control of Agent communicate with each other, to develop its own distribution network without task Scheduler power regulation and reactive power management, as well as step-type wind power and reactive power compensation in the process of adjusting the distribution of power. Centralized Control Agent in receiving feedback after the members of its jurisdiction, all information processing. In process control, and if a large load changes occur within a longer period of time, reactive power balance issues within the larger area, then put MCR under centralized control, in order to achieve decentralized wind power reactive control problems fast processing; If a smaller load changes in a short period of time, in a small area of reactive power control problem, the direct control of its distribution area where Agent themselves. Distributed Control Agent after receiving the program, through the Agent on each node within the jurisdiction of the operation and the surrounding Agent comprehensive analysis of distributed control operation, the development of specific programs Node Agent reactive power regulation and reactive power required size adjustment notify the appropriate amount distributed control Agent. Distributed control at the receiving node Agent Agent after adjustment notice, according to their specific MCR operating within its jurisdiction, while running a real-time feedback to adjust the distribution of control Agent, then followed up the level of centralized control is passed Feedback Agent information, and wait for the next coordinate.

Such a model to achieve the coordination and management of centralized control and distributed control, make full use Muti-Agent system autonomy, interaction and initiative features optimized by direct action on the node reactive power devices implement the entire reactive power distribution grid, this reactive power control to balance the advantages and disadvantages of the structure of centralized and distributed two structures, while maintaining their independence under premise Agent, through the same layer or between adjacent levels of a certain amount of data to ensure mutual contact each Agent decision is reasonable, adapted to large-scale distribution of complex reactive power control.

\section{MARKOV GAMES OF MAS}

The $\Pi$-type equivalent circuit of power line is shown in Fig .3, where $Z=R+j X, Y=G+j B$ is the each phase of the power line impedance and admittance, $\dot{U}$ is phase voltage, $\tilde{S}$ is a single phase power.

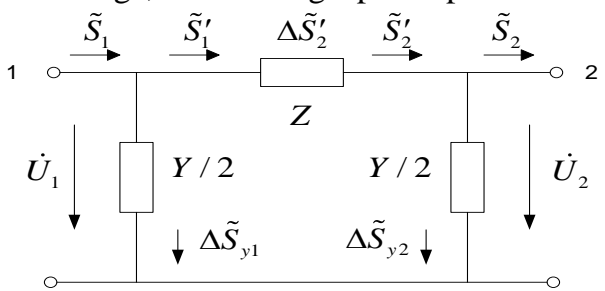

Figure 3. Equivalent Circuit of Power Line 
Set terminal voltage of $\dot{U}_{2}$, the end of the power $\tilde{S}_{2}=P_{2}+j Q_{2}$, the end of the line power loss admittance slip $\Delta \dot{S}_{y 2}$ as follows:

$$
\Delta \dot{S}_{y 2}=\frac{1}{2}(G-j B) U_{2}^{2}=\Delta P_{y 2}-J \Delta Q_{y 2}
$$

$\tilde{S}_{2}^{\prime}$ is the impedance of the end of the power:

$$
\tilde{S}_{2}^{\prime}=\tilde{S}_{2}+\Delta \dot{S}_{y 2}=P_{2}^{\prime}+j Q_{2}^{\prime}
$$

When a power line transmission, the current will produce a voltage loss in the line impedance. The voltage vector of power line is shown in Fig .4.

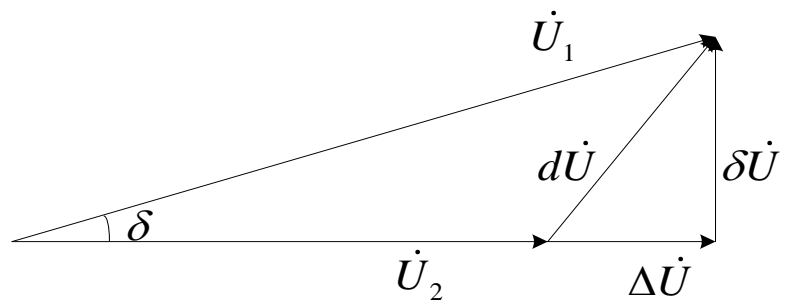

Figure 4. Voltage Vector of Power Line

$$
\dot{U}_{1}=\left(U_{2}+\frac{P_{2}^{\prime} R+Q_{2}^{\prime} X}{U_{2}}\right)+j\left(\frac{P_{2}^{\prime} X-Q_{2}^{\prime} R}{U_{2}}\right)
$$

Let $\Delta U=\frac{P_{2}^{\prime} R+Q_{2}^{\prime} X}{U_{2}}, \delta U=\frac{P_{2}^{\prime} X-Q_{2}^{\prime} R}{U_{2}}$,

(3) can be written as

$$
\dot{U}_{1}=\left(U_{2}+\Delta U\right)+j \delta U
$$

Fig .2 shows the voltage loss is

$$
d U=\sqrt{(\Delta U)^{2}+(\delta U)^{2}}
$$

Where $d U$ is vector difference of The first terminal voltage $\dot{U}_{1}$ and the terminal voltage $\dot{U}_{2}$, That is current in the line impedance voltage drop, voltage loss for the first and last line voltage across the value difference. $\Delta \dot{U}$ is projected of $d \dot{U}$ in the direction $\dot{U}_{2}$, called the vertical component of the voltage drop. $\delta \dot{U}$ is projected of $d \dot{U}$ in the vertical direction $\dot{U}_{2}$, called transverse component of the voltage drop.

In general the power system, $U_{2}+\Delta U>>\delta U$, so it can be Negligible, which can be obtained (6).

$$
U_{1}=U_{2}+\frac{P_{2}^{\prime} R+Q_{2}^{\prime} X}{U_{2}}
$$

In general $\mathrm{HV}$ and $\mathrm{EHV}$ power grid, due to a larger cross-section of transmission line conductors, line reactance is much greater than the resistance, (6) is expressed as (7).

$$
U_{1}-U_{2}=d U=\frac{Q_{2}^{\prime} X}{U_{2}}
$$

In the case of the grid structure and the voltage determined by the reactive power of the system plays a decisive influence on the voltage level, the voltage loss of main line and Reactive power of line transmission delivered is proportional, the voltage amplitude of the reactive power flow of the high voltage node low amplitude nodes [12]. Because in general the grid transformer branch, the resistance value is far less than the series reactance, so reactive power is the main factor causing the loss of branch voltage transformer. Therefore, reactive power grid optimization, changing the distribution of reactive power, a reasonable choice of local reactive balance in order to reduce the flow of reactive power on the line, can effectively reduce the network voltage loss, ensure stability of the system.

Reactive power optimization mathematical models generally include the objective function, power constraint equations and variables constrained conditions. Generic model can be expressed as the following:

$$
\left\{\begin{array}{c}
\min f(u, x) \\
\text { s.t.g }(u, x)=0 \\
h(u, x) \leq 0
\end{array}\right.
$$

Where $u$ is the control variables, including the generator terminal voltage, load tap variable gear ratio and shunt capacitors; $x$ is the state variables, including the voltage amplitude of node $P Q$ and reactive power of node $P V, f(u, x)$ is no power optimization objective function; $g(u, x)$ is the power constraint equation, $h(u, x)$ is variable constraints.

Reactive power optimization objective function, depending on the focus of specific optimization can be divided into multiple, often choose system minimum loss of active network as objective function:

$$
\begin{gathered}
F=\min \sum_{k=1}^{n} P_{\text {kloss }} \\
=\min \sum_{k=1}^{n} G_{k(i, j)}\left[U_{i}^{2}+U_{j}^{2}-2 U_{i} U_{j} \cos \left(\delta_{i}-\delta_{j}\right)\right]
\end{gathered}
$$

Wherein $n$ is the aggregate expenditure of large ones network; $G_{k(i, j)}$ is the conductance of branch $i-j ; U_{i}$ 
and $U_{j}$ are the voltages of node $i$ and $j ; \delta_{i}$ and $\delta_{j}$ are the voltage phase angle of node $i$ and $j$.

Reactive power optimization problem in variables divided in control variables and state variables. Select the generator terminal voltage is usually $U_{G}$, OLTC transformer ratio $T_{t}$ and shunt capacitance of the capacitor $Q_{C}$ as the control variable, load node voltage $U_{D}$ and generator reactive power $Q_{G}$ as the state variables.

Control variable inequality constraints as the following:

$$
\left\{\begin{array}{cc}
U_{G i, \text { min }} \leq U_{G i} \leq U_{G i, \max } & i \in N_{G} \\
T_{t j \text { min }} \leq T_{t j} \leq T_{t j, \text { max }} & j \in N_{T} \\
Q_{C k, \text { min }} \leq Q_{C k} \leq Q_{C k, \text { max }} & k \in N_{C}
\end{array}\right.
$$

Where $U_{G i}$ is the voltage of generator node $i$, $U_{G i \text {,min }}$ and $U_{G i \text {, max }}$ are the lower limit and upper limit of the node voltage, $N_{G}$ is a generator set of nodes; $T_{t j}$ is ratio of load tap transformer $j, T_{t j \text {,min }}$ and $T_{t j \text {,max }}$ are ratio of the transformer lower limit and upper limit, $N_{T}$ is the set of load tap; $Q_{C k}$ is reactive power compensation capacity of capacitor $k ; Q_{C k \text {,min }}$ and $Q_{C k \text {, max }}$ are lower limit and upper limit of reactive power compensation capacitor, $N_{C}$ is the reactive power compensation capacitor node collection.

Inequality constraints state variables as the following:

$$
\begin{cases}Q_{G i, \min } \leq Q_{G i} \leq Q_{G i, \max } & i \in N_{G} \\ U_{D j, \min } \leq U_{D j} \leq U_{D j, \max } & j \in N_{D}\end{cases}
$$

Where $Q_{G i}$ is the generator reactive power output of node $i, Q_{G i \text {, min }}$ and $Q_{G i \text {, max }}$ are the lower limit and upper limit of generator reactive power, $N_{G}$ is a generator set of nodes; $U_{D j}$ is voltage of load node $j, U_{D j \text {, min }}$ and $U_{D j, \max }$ are lower limit and upper limit of load node voltage, $N_{D}$ is the load node collection.

\section{CONCLUSION}

Muti-Agent technology is mainly a group of autonomous Agent research in distributed an open and dynamic environment, through interactive cooperation, competition, negotiation and other complex control intelligent behavior or task to solve, because it better reflects human social intelligence, more suitable open, dynamic social environment, Muti-Agent system can solve some of the traditional methods can not solve the problem, such as an open, dynamic and complex nature, etc., especially suitable for highly open, loosely coupled network environment. Meanwhile, in a complex control system, in accordance with the system features and physical characteristics, the system control structure is divided into functionally independent, with autonomy, flexibility, reactivity and sociality Agent, through the communication between the individual and the Agent interaction between the individual controllers to achieve coordinated action in order to ensure the best overall system control.

\section{ACKNOWLEDGMENT}

The authors gratefully acknowledge the contribution of co-workers and reviewers' comments.

\section{REFERENCES}

[1] G. R. M. COSTA and C. E. U. COSTA, "Improved Newton method for optimal power flow problem," Electrical Power \& Energy Systems, vol. 22, pp. 459-462, Dec. 2000.

[2] M. B. Liu,Y. Cheng and S. H. Lin, "Comparative studies of interior-point linear and nonlinear programming algorithms for reactive power optimization," Automation of Electric Power Systems, vol. 26, pp. 22-26, Oct. 2002.

[3] J. T. Ma and Y. H. Yang, "Application of genetic algorithms in reactive power optimization," Proceeding of the CSEE, pp. 347353, Dec. 1995.

[4] J. Hu and M. P. Wellman, "Multiagent reinforcement learning: theoretical framework and an algorithm," Proceedings of the Fifteenth International Conference on Machine Learning, Morgan Kaufmann, 1998 , pp.62-68.

[5] G. Wagner, "Agent-Oriented Analysis and Design of Organi zational Information System," Proceedings of Fourth IEEE International Baltic Workshop on Database and Information Systems, pp. 192-198, May 2000.

[6] K. Erol, L. Lang and R. Levy, "Designing Agent form Reusable Components," The fourth international conference on Autonomous agents, pp. 74-78, Dec. 2000.

[7] G. D. Liao and X. R. Wang, "On-Line identification method for critical buses of power system voltage stability," Journal of Southwest Jiaotong University, vol. 44, pp. 244-248, Dec. 2009.

[8] W. B. Arthur, "Complexity in economic and financial markets," Complexity, vol. 132, pp. 489-494, Oct. 1995.

[9] G. Zhang, "Peter Time series forcasting using a hybrid ARIMA and nature network model,"Neural computing, vol. 12, pp. 159175,Dec. 2003.

[10] M. L. Littman, "Markov games as a framework formult-i agent reinforcement learning," Proceedings of the Eleventh International Conference on Machine Learning, pp. 157-163, Oct. 1994.

[11] M. L. Littman, "Value-function reinforcement learning in Markov games," Journal of Cognitive Systems Research, vol. 12, pp. 1-12, Jan. 2001.

[12] R. sharma and M. GoPal, "Markov Game Controller Degin algorithm, 'International journal of Computation Intelligence, vol. 3, pp. 1304-1318,Oct. 2006. 